Research Article

\title{
On the Incompressible Limit for the Compressible Flows of Liquid Crystals under Strong Stratification on Bounded Domains
}

\author{
Young-Sam Kwon \\ Department of Mathematics, Dong-A University, Busan 604-714, Republic of Korea \\ Correspondence should be addressed to Young-Sam Kwon; ykwon@dau.ac.kr \\ Received 10 October 2012; Accepted 12 January 2013 \\ Academic Editor: Norio Yoshida
}

Copyright (C) 2013 Young-Sam Kwon. This is an open access article distributed under the Creative Commons Attribution License, which permits unrestricted use, distribution, and reproduction in any medium, provided the original work is properly cited.

We study the incompressible limit of weak solutions for the compressible flows of liquid crystals under strong stratification on bounded domains.

\section{Introduction}

Liquid crystals flows can be found in the natural world and in technological applications with a variety of examples such as many proteins, cell membranes, and solutions of soap and various related detergents, as well as the tobacco mosaic virus. We here consider the compressible flows of liquid crystals:

$$
\begin{gathered}
\partial_{t} \varrho+\operatorname{div}_{x}(\varrho \mathbf{u})=0, \\
\partial_{t}(\varrho \mathbf{u})+\operatorname{div}_{x}(\varrho \mathbf{u} \otimes \mathbf{u})+\nabla_{x} p(\varrho)=\mu \Delta \mathbf{u}, \\
-v \operatorname{div}_{x}\left(\nabla \mathbf{d} \otimes \nabla \mathbf{d}-\left(\frac{1}{2}|\nabla \mathbf{d}|^{2}+F(\mathbf{d})\right) \mathbb{a}_{3}\right)+\varrho \nabla G, \\
\partial_{t} \mathbf{d}+\mathbf{u} \cdot \nabla \mathbf{d}=\theta(\Delta \mathbf{d}-f(\mathbf{d})), \quad \mathbf{d}(0, x)=\mathbf{d}_{0}(x),
\end{gathered}
$$

where $\mathbf{u}$ is the vector field, $\varrho$ is the density, $\mathbf{d}$ is the direction field for the averaged macroscopic molecular orientations, $G$ is a given potential, and $\mu, v, \theta$ are viscosities. The smooth vector $f(\mathbf{d})$ and the smooth scalar function $F(\mathbf{d})$ are related by

$$
f(\mathbf{d})=\nabla_{\mathbf{d}} F(\mathbf{d}),
$$

where $f(\mathbf{d})$ represents the penalty function and $F(\mathbf{d})$ is the bulk part of the elastic energy verifying: there exists $C>0$ such that if $|\mathbf{d}| \geq C$,

$$
f(\mathbf{d}) \cdot \mathbf{d} \geq 0 .
$$

Note that the condition (5) is required for the global weak solution. We can see an example as follows:

$$
F(\mathbf{d})=\frac{1}{4 \sigma_{0}^{2}}\left(|\mathbf{d}|^{2}-1\right)^{2}, \quad f(\mathbf{d})=\frac{1}{2 \sigma_{0}^{2}}\left(|\mathbf{d}|^{2}-1\right) \mathbf{d},
$$

where $\sigma_{0}$ is a constant. Finally, the pressure $p$ is defined as follows

$$
\begin{gathered}
p(0)=0, \quad p \in C^{2}[0, \infty), \quad p^{\prime}(r)>0 \quad \forall r \geq 0, \\
\lim _{r \rightarrow \infty} \frac{p^{\prime}(r)}{r^{\gamma-1}}=q>0,
\end{gathered}
$$

and the function $\widetilde{\varrho}$ is the unique positive solution of the following problem:

$$
p_{0} \nabla \widetilde{\varrho}=\widetilde{\varrho} \nabla G, \quad p_{0}=p^{\prime}(0) .
$$

We use the following scaling used in Feireisl et al. [1] and Wang and $\mathrm{Yu}[2]$ :

$$
t \approx \epsilon t, \quad x \approx x, \quad Q \approx \varrho_{\epsilon}, \quad \mathbf{u} \approx \epsilon \mathbf{u}_{\epsilon}, \quad \mathbf{d} \approx \mathbf{d}_{\epsilon},
$$

and for the viscosity coefficients,

$$
\mu \approx \epsilon \mu_{\epsilon}, \quad v \approx \epsilon^{2} v_{\epsilon}, \quad \theta \approx \epsilon \theta_{\epsilon},
$$

with the convergence of the viscosity coefficients

$$
\mu_{\epsilon} \longrightarrow \mu, \quad v_{\epsilon} \longrightarrow v, \quad \theta_{\epsilon} \longrightarrow \theta .
$$


Finally for the pressure, we use

$$
p_{\epsilon}(\varrho)=\frac{1}{\epsilon^{\alpha}} p\left(\epsilon^{\alpha} \varrho\right), \quad \alpha \in(2,3) .
$$

Following the above scalings, the system (1)-(7) reads

$$
\begin{gathered}
\partial_{t} \varrho_{\epsilon}+\operatorname{div}_{x}\left(\varrho_{\epsilon} \mathbf{u}_{\epsilon}\right)=0 \\
\partial_{t}\left(\varrho_{\epsilon} \mathbf{u}_{\epsilon}\right)+\operatorname{div}_{x}\left(\varrho_{\epsilon} \mathbf{u}_{\epsilon} \otimes \mathbf{u}_{\epsilon}\right)+\frac{1}{\epsilon^{2}} \nabla_{x} p_{\epsilon}\left(\varrho_{\epsilon}\right) \\
=\mu_{\epsilon} \Delta \mathbf{u}_{\epsilon}-v_{\epsilon} \operatorname{div}_{x}\left(\nabla \mathbf{d}_{\epsilon} \otimes \nabla \mathbf{d}_{\epsilon}-\left(\frac{1}{2}\left|\nabla \mathbf{d}_{\epsilon}\right|^{2}+F\left(\mathbf{d}_{\epsilon}\right)\right) \rrbracket_{3}\right) \\
+\frac{1}{\epsilon^{2}} \varrho_{\epsilon} \nabla G, \\
\partial_{t} \mathbf{d}_{\epsilon}+\mathbf{u} \cdot \nabla \mathbf{d}_{\epsilon}=\theta_{\epsilon}\left(\Delta \mathbf{d}_{\epsilon}-f\left(\mathbf{d}_{\epsilon}\right)\right) .
\end{gathered}
$$

We now notice that the global-in-time existence solutions for system ((1)-(3)) have been studied by Wang and Yu [3] and Liu and Qing [4]. For the case of $\mathbf{d}=0$, incompressible limit problems have been investigated by many authors, starting with the work by Klainerman and Majda [5] for the Euler equations and Lions and Masmoudi [6] for the isentropic Navier Stokes equations. Similar results in the spirit of the analysis presented by Lions and Masmoudi [6] are the recent progress by Feireisl and Novotný $[7,8]$ and Kwon and Trivisa [9] for the full Navier-Stokes Fourier system. For the liquid crystals, there is one recent progress by Wang and $\mathrm{Yu}$ [2] based on the spectral analysis and Duhamel's principle to control difficulties arising in the boundary of bounded domains. There are some results of incompressible limit problems of Navier Stoke Fourier system under strong stratification by Feireisl and Novotný [7] on bounded domain and by Feireisl et al. on unbounded domains, which is extended to full magnetohydrodynamic flows on bounded domain by Novotný et al. [10] and by Lee et al. [11].

For this kind of application, the polymer precursor leads to a stratified structure of a solidified film of polymer and so we have a natural question: is such fluid almost incompressible such that it is strongly stratified when $\epsilon \rightarrow 0$ as the different models of compressible fluid? In this paper, we derive the rigorous result of the incompressible limit of the flow of liquid crystals with the similar idea used in the previous results $[7,10-12]$. Formally, we will investigate the limit

$$
\varrho_{\epsilon} \longrightarrow \tilde{\varrho}, \quad \mathbf{u}_{\epsilon} \longrightarrow \mathbf{U}, \quad \mathbf{d}_{\epsilon} \longrightarrow \mathbf{d}
$$

as $\epsilon$ tends to 0 in the suitable sense such that the given limit $\{\widetilde{\varrho}, \mathbf{U}, \mathbf{d}\}$ represents a solution of the following system:

$$
\begin{gathered}
\operatorname{div}_{x}(\widetilde{\varrho} \mathbf{U})=0, \\
\partial_{t}(\widetilde{\varrho} \mathbf{U})+\operatorname{div}_{x}(\widetilde{\varrho} \mathbf{U} \otimes \mathbf{U})+\nabla_{x} P \\
=\mu \Delta \mathbf{U}-\left(\frac{1}{2}|\nabla \mathbf{d}|^{2}+F(\mathbf{d}) \square_{3}\right), \\
\partial_{t} \mathbf{d}+\mathbf{U} \cdot \nabla \mathbf{d}=\theta(\Delta \mathbf{d}-f(\mathbf{d})) .
\end{gathered}
$$

Finally, in this paper, we will use the many parts of the presentation of Feireisl and Novotný [7] and Feireisl et al. [12] without modification.

The outline of this paper is as follows: In Section 2 we present two initial-boundary-value problems and introduce the notion of weak solutions for the compressible fluid of liquid crystals. In Section 3 we present the main results of the article on the low Mach number problems under strong stratification on bounded domains. In Section 4, we present the proof of the low Mach number problem for bounded domains.

\section{Weak Solutions}

2.1. An Initial-Boundary-Value Problem. Let $\Omega \subset \mathbb{R}^{3}$ be a bounded domain with the boundary of class $C^{\infty}$

$$
\left.\mathbf{u} \cdot \mathbf{n}\right|_{\partial \Omega}=0
$$

where $\mathbf{n}$ stands for the outer normal vector. We also propose the boundary condition on the direction vector $\mathbf{d}$

$$
\left.\mathbf{d}\right|_{\partial \Omega}=\mathbf{d}_{0}
$$

Notice that Wang and Yu showed the global weak solution of the system (1)-(3) with the Dirichlet boundary condition on bounded domains but there will be no problem with the boundary conditions (18) and (19) for existence result.

\subsection{Weak Solutions}

Definition 1. We say that a quantity $\{\varrho, \mathbf{u}, \mathbf{d}\}$ is a weak solution of the compressible flows of liquid crystals (13)-(15) supplemented with the initial data $\left\{\varrho_{0}, \mathbf{u}_{0}, \mathbf{d}_{0}\right\}$ provided that the following hold.

(i) The density $\varrho$ is a nonnegative function, $\varrho \in$ $L^{\infty}\left(0, T ; L^{\gamma}(\Omega)\right)$, the velocity field $\mathbf{u} \in L^{2}\left(0, T ; H^{1}(\Omega\right.$; $\left.\left.\mathbb{R}^{3}\right)\right), \varrho|\mathbf{u}|^{2} \in L^{\infty}\left(0, T ; L^{1}(\Omega)\right)$, and $\mathbf{u}$ represents a renormalized solution of (1) on a time-space cylinder $(0, T) \times \Omega$, that is, the integral identity

$$
\begin{gathered}
\int_{0}^{T} \int_{\Omega}\left(\varrho B(\varrho) \partial_{t} \varphi+\varrho B(\varrho) \mathbf{u} \cdot \nabla_{x} \varphi\right. \\
\left.-b(\varrho) \operatorname{div}_{x} \mathbf{u} \varphi\right) \mathrm{d} x \mathrm{~d} t \\
=-\int_{\Omega} \varrho_{0} B\left(\varrho_{0}\right) \varphi(0, \cdot) \mathrm{d} x
\end{gathered}
$$

holds for any test function $\varphi \in \mathscr{D}([0, T) \times \bar{\Omega})$ and any $b$ such that

$$
\begin{gathered}
b \in L^{\infty} \cap C[0, \infty), \\
B(\varrho)=B(1)+\int_{1}^{\varrho} \frac{b(z)}{z^{2}} \mathrm{~d} z .
\end{gathered}
$$


(ii) The balance of momentum holds in distributional sense, namely,

$$
\begin{gathered}
\int_{0}^{T} \int_{\Omega}\left(\varrho \mathbf{u} \cdot \partial_{t} \vec{\varphi}+\varrho \mathbf{u} \otimes \mathbf{u}\right. \\
: \nabla_{x} \vec{\varphi}+\frac{1}{\epsilon^{2}} p_{\epsilon}(\varrho) \operatorname{div}_{x} \vec{\varphi}-\nu_{\epsilon} \Pi \\
: \nabla \vec{\varphi}+\mu_{\epsilon} \nabla \mathbf{u} \\
\left.: \nabla \vec{\varphi}+\frac{1}{\epsilon^{2}} \nabla G \cdot \vec{\varphi}\right) \mathrm{d} x \mathrm{~d} t \\
=-\int_{\Omega} \varrho_{0} \mathbf{u}_{0} \cdot \vec{\varphi}(0, \cdot) \mathrm{d} x
\end{gathered}
$$

for any test function $\vec{\varphi} \in \mathscr{D}\left([0, T) ; \mathscr{D}\left(\bar{\Omega} ; \mathbb{R}^{3}\right)\right)$ satisfying $\left.\vec{\varphi} \cdot \mathbf{n}\right|_{\partial \Omega}=0$, where $\Pi$ is defined by

$$
\Pi=\nabla \mathbf{d} \otimes \nabla \mathbf{d}-\left(\frac{1}{2}|\nabla \mathbf{d}|^{2}+F(\mathbf{d})\right) \mathbb{q}_{3} .
$$

(iii) The total energy of the system holds:

$$
\begin{aligned}
E(t)+\int_{0}^{t} \int_{\Omega}\left(\mu_{\epsilon}|\nabla \mathbf{u}|^{2}\right. & \\
& \left.\quad+v_{\epsilon} \theta_{\epsilon}|\Delta \mathbf{d}-f(\mathbf{d})|^{2}\right) \mathrm{d} x \mathrm{~d} t \\
& \leq E(0)
\end{aligned}
$$

holds for a.e. $t \in(0, T)$, where

$$
\begin{gathered}
E(t)=\int_{\Omega}\left(\frac{1}{2} \varrho|\mathbf{u}|^{2}+\frac{1}{\epsilon^{2}} H_{\epsilon}(\varrho)+\frac{v_{\epsilon}}{2}|\mathbf{d}|^{2}\right. \\
\left.+v_{\epsilon} F(\mathbf{d})-\varrho G\right) \mathrm{d} x
\end{gathered}
$$

with

$$
H_{\epsilon}(\varrho)=\varrho \int_{1}^{\varrho} \frac{p_{\epsilon}(\varrho)}{z^{2}} \mathrm{~d} z
$$

(iv) The equation of direction field verifies

$$
\begin{aligned}
& \int_{0}^{T} \int_{\Omega} \mathbf{d} \cdot \partial_{t} \vec{\varphi} \mathrm{d} x \mathrm{~d} t \\
&+\int_{0}^{T} \int_{\Omega}(\mathbf{u} \cdot \mathbf{d}) \operatorname{div}_{x} \vec{\varphi}+(\nabla \mathbf{u} \cdot \mathbf{d}) \cdot \vec{\varphi} . \\
& \quad-\theta(\nabla \mathbf{d}+f(\mathbf{d})) \cdot \vec{\varphi} \mathrm{d} x \mathrm{~d} t=0
\end{aligned}
$$

for all $\vec{\varphi} \in[\mathscr{D}([0, T) \times \Omega)]^{3}$.

Let us now discuss the static states which are solutions of system (13)-(15) with vanishing velocity field. In the present setting, the positive density $\widetilde{\varrho}_{\epsilon}$ must satisfy

$$
\nabla p_{\epsilon}\left(\widetilde{\varrho}_{\epsilon}\right)=\widetilde{\varrho}_{\epsilon} \nabla G, \quad p_{0}=p^{\prime}(0) .
$$

From the statistic equation (28), we can easily derive

$$
\begin{gathered}
p_{0} \log (\widetilde{\varrho}(x))+Q\left(\epsilon^{\alpha} \widetilde{\varrho}(x)\right)-Q\left(\epsilon^{\alpha} \varrho_{\infty}\right) \\
=G(x)+p_{0} \log \left(\varrho_{\infty}\right)
\end{gathered}
$$

with

$$
Q^{\prime}(r)= \begin{cases}\frac{P^{\prime}(r)-p_{0}}{r}, & \text { if } r>0, \\ P^{\prime \prime}(0), & \text { if } r=0 .\end{cases}
$$

We now introduce the weak solutions of the target system.

Definition 2. A couple $\{\widetilde{\varrho}, \mathbf{U}, \mathbf{d}\}$ is said to be a weak solution of the target system of the compressible flows of liquid crystals with the potential $G \in W^{1, \infty}(\Omega), \nabla G \in L^{1}(\Omega)$, supplemented with the boundary conditions

$$
\mathbf{U} \cdot \mathbf{n}=0, \quad \mathbf{d}=\mathbf{d}_{0},
$$

on $\partial \Omega$ which belongs to $C^{\infty}$, and the initial conditions

$$
\mathbf{U}(0, \cdot)=\mathbf{U}_{0}, \quad \mathbf{d}(0, \cdot)=\mathbf{d}_{0}
$$

with $\mathbf{U} \in L^{2}(\Omega), \mathbf{d}_{0} \in H^{1}(\Omega),\left.\mathbf{d}_{0}\right|_{\partial \Omega} \in H^{3 / 2}(\Omega)$ if the following conditions hold:

(i) $\mathbf{U} \in L^{\infty}\left(0, T ; L^{2}\left(\Omega ; \mathbb{R}^{3}\right)\right) \cap L^{2}\left(0, T ; H^{1}\left(\Omega ; \mathbb{R}^{3}\right)\right)$, $\operatorname{div}_{x}(\widetilde{U})=0$ a.e. on $(0, T) \times \Omega,\left.\mathbf{U} \cdot \mathbf{n}\right|_{\partial \Omega}=0$ in the sense of traces, and the integral identity

$$
\begin{aligned}
\int_{0}^{T} \int_{\Omega}\left(\widetilde{\varrho} \cdot \partial_{t} \varphi+(\widetilde{U} \otimes \mathbf{U})\right. \\
\left.\quad: \nabla_{x} \varphi-\mu \nabla_{x} \mathbf{U}: \nabla_{x} \varphi\right) \mathrm{d} x \mathrm{~d} t \\
=-\int_{0}^{T} \int_{\Omega}(\nabla \mathbf{d} \otimes \nabla \mathbf{d}): \nabla \varphi \mathrm{d} x \mathrm{~d} t \\
-\int_{\Omega} \mathbf{U} \cdot \varphi(0, \cdot) \mathrm{d} x
\end{aligned}
$$

holds for any test function

$$
\begin{gathered}
\varphi \in \mathscr{D}\left((0, T) \times \Omega ; \mathbb{R}^{3}\right), \\
\operatorname{div}_{x} \varphi=0 \quad \text { in } \Omega,\left.\quad \varphi \cdot \mathbf{n}\right|_{\partial \Omega}=0,
\end{gathered}
$$

(ii) $\mathbf{d} \in L^{2}\left(0, T ; H^{2}\left(\Omega ; \mathbb{R}^{3}\right)\right) \cap L^{\infty}\left(0, T ; H^{1}\left(\Omega ; \mathbb{R}^{3}\right)\right)$, and the integral identity

$$
\begin{aligned}
& \int_{0}^{T} \int_{\Omega} \mathbf{d} \cdot \partial_{t} \vec{\varphi} \mathrm{d} x \mathrm{~d} t \\
&+\int_{0}^{T} \int_{\Omega}\left((\mathbf{U} \cdot \mathbf{d}) \operatorname{div}_{x} \vec{\varphi}+(\nabla \mathbf{U} \cdot \mathbf{d}) \cdot \vec{\varphi}\right. \\
&-\theta(\nabla \mathbf{d}+f(\mathbf{d})) \cdot \vec{\varphi}) \mathrm{d} x \mathrm{~d} t=0
\end{aligned}
$$

holds for all $\vec{\varphi} \in[\mathscr{D}([0, T) \times \Omega)]^{3}$. 


\section{Main Results}

In this section we mention the main result as follows.

Theorem 3. Let $\Omega \subset \mathbb{R}^{3}$ be a bounded domain with a boundary of class $C^{\infty}$ and $\left\{\varrho_{\epsilon}, \mathbf{u}_{\epsilon}, \mathbf{d}_{\epsilon}\right\}$ a family of weak solutions to the compressible of liquid crystals system verifying (5) in the sense of Definition 1 with $G \in W^{1, \infty}(\Omega), \nabla G \in L^{1}(\Omega)$. Assume that the initial condition is as follows:

$$
\begin{array}{r}
\left\{\varrho_{0, \epsilon}^{(1)}\right\}_{\epsilon>0} \text { bounded in }\left(L^{2} \cap L^{\infty}\right)(\Omega), \\
\text { with } \varrho_{0, \epsilon}^{(1)}:=\frac{\varrho_{0, \epsilon}-\tilde{\varrho}}{\epsilon}, \\
\left\{\mathbf{u}_{0, \epsilon}\right\}_{\epsilon>0} \text { bounded in }\left(L^{2} \cap L^{\infty}\right)\left(\Omega ; \mathbb{R}^{3}\right), \\
\left\{\mathbf{d}_{0, \epsilon}\right\}_{\epsilon>0} \text { bounded in }\left(L^{2} \cap L^{\infty}\right)\left(\Omega ; \mathbb{R}^{3}\right) .
\end{array}
$$

Then, up to subsequence,

$$
\begin{array}{cc}
\varrho_{\epsilon} \longrightarrow \text { @a.e. } & \text { in }(0, T) \times \Omega, \\
\mathbf{u}_{\epsilon} \longrightarrow \mathbf{U} \text { a.e. } & \text { in }(0, T) \times \Omega, \\
\mathbf{d}_{\epsilon} \longrightarrow \mathbf{d} \text { a.e. } & \text { in }(0, T) \times \Omega,
\end{array}
$$

where $\{\tilde{\varrho}, \mathbf{U}, \mathbf{d}\}$ solves a weak solution of the incompressible flows of liquid crystals in the sense of Definition 2 with the boundary condition $\left.\mathbf{U} \cdot \mathbf{n}\right|_{\partial \Omega}=0$ and the initial data

$$
\mathbf{U}(0)=\mathbf{P}_{\widetilde{\varrho}}\left[\mathbf{U}_{0}\right], \quad \mathbf{d}(0, \cdot)=\mathbf{d}_{0},
$$

where the Helmholtz projection $\mathbf{P}_{\widetilde{\varrho}}=\mathbf{I}-\mathbf{Q}_{\widetilde{\varrho}}$ and $\mathbf{Q}_{\widetilde{\varrho}}$ is defined in (81).

\section{Proof of Theorem 3}

4.1. Uniform Bounds. In this section we are going to derive some estimates on the sequence $\left\{\varrho_{\epsilon}, \mathbf{u}_{\epsilon}, \mathbf{d}_{\epsilon}\right\}_{\epsilon>0}$. Multiplying (13) by $H^{\prime}\left(\varrho_{\epsilon}\right)-H^{\prime}\left(\widetilde{\varrho}_{\epsilon}\right)$ and adding the energy inequality (24), it follows that

$$
\begin{aligned}
\int_{\Omega}( & \frac{1}{2} \varrho_{\epsilon}\left|\mathbf{u}_{\epsilon}\right|^{2}+\frac{1}{\epsilon^{2}}\left(H_{\epsilon}\left(\varrho_{\epsilon}\right)-H_{\epsilon}\left(\widetilde{\varrho}_{\epsilon}\right)-H_{\epsilon}^{\prime}\left(\widetilde{\varrho}_{\epsilon}\right)\left(\varrho_{\epsilon}-\widetilde{\varrho}_{\epsilon}\right)\right) \\
& \left.+\frac{v_{\epsilon}}{2}\left|\mathbf{d}_{\epsilon}\right|^{2}+v_{\epsilon} F\left(\mathbf{d}_{\epsilon}\right)\right) \mathrm{d} x \\
& +\int_{0}^{t} \int_{\Omega}\left(\mu_{\epsilon}\left|\nabla \mathbf{u}_{\epsilon}\right|^{2}+v_{\epsilon} \theta_{\epsilon}\left|\Delta \mathbf{d}_{\epsilon}-f\left(\mathbf{d}_{\epsilon}\right)\right|^{2}\right) \mathrm{d} x \mathrm{~d} t \leq E_{0, \epsilon},
\end{aligned}
$$

where

$$
\begin{aligned}
E_{0, \epsilon}=\int_{\Omega}\left(\frac{1}{2} \varrho_{0, \epsilon}\left|\mathbf{u}_{0, \epsilon}\right|^{2}\right. & \\
& +\frac{1}{\epsilon^{2}}\left(H_{\epsilon}\left(\varrho_{0, \epsilon}\right)-H_{\epsilon}\left(\widetilde{\varrho}_{\epsilon}\right)-H^{\prime}\left(\widetilde{\varrho}_{\epsilon}\right)\left(\varrho_{0, \epsilon}-\widetilde{\varrho}_{\epsilon}\right)\right) \\
& \left.+\frac{v_{\epsilon}}{2}\left|\mathbf{d}_{0, \epsilon}\right|^{2}+v_{\epsilon} F\left(\mathbf{d}_{0, \epsilon}\right)\right) \mathrm{d} x .
\end{aligned}
$$

For convenient presentation, we introduce the set of the essential and residual values

$$
g=[g]_{\mathrm{ess}}+[g]_{\mathrm{res}}
$$

where $[g]_{\mathrm{ess}}=\chi\left(\varrho_{\epsilon}\right) g,[g]_{\mathrm{res}}=\left(1-\chi\left(\varrho_{\epsilon}\right)\right) g$ and $\chi$ is defined as follows:

$$
\chi(r)=1 \quad \forall r \in\left[\frac{\underline{\varrho}}{2,2 \bar{\varrho}}\right], \quad \chi(r)=0 \text { otherwise, }
$$

where $\widetilde{\varrho}_{\epsilon}$ is the solution of (28) and

$$
\underline{\varrho}=\inf _{\epsilon>0} \inf _{x \in \Omega} \widetilde{\varrho}_{\epsilon}(x), \quad \bar{\varrho}=\sup _{\epsilon>0} \sup _{x \in \Omega} \widetilde{\varrho}_{\epsilon}(x) .
$$

Notice that two assumptions of pressure in (7) and (12) imply that $H_{\epsilon}$ is a strict convex. Thus, thanks to (36), we get that $E_{0, \epsilon}$ is uniformly bounded for $\epsilon \rightarrow 0$. Consequently, from the energy balance (40), we obtain

$$
\begin{aligned}
& \text { ess } \sup _{t \in(0, T)}\left\|\sqrt{\varrho_{\epsilon}} \mathbf{u}_{\epsilon}(t)\right\|_{L^{2}\left(\Omega ; \mathbb{R}^{3}\right)} \leq C, \\
& \text { ess } \sup _{t \in(0, T)}\left\|\mathbf{d}_{\epsilon}(t)\right\|_{L^{2}\left(\Omega ; \mathbb{R}^{3}\right)} \leq C \\
& \text { ess } \sup _{t \in(0, T)}\left\|\left(\Delta \mathbf{d}_{\epsilon}-f\left(\mathbf{d}_{\epsilon}\right)\right)(t)\right\|_{L^{2}\left(\Omega ; \mathbb{R}^{3}\right)} \leq C, \\
& \left\|\nabla_{x} \mathbf{u}_{\epsilon}\right\|_{L^{2}((0, T) \times \Omega)} \leq C, \\
& \text { ess } \sup _{t \in(0, T)}\left\|\left[H_{\epsilon}\left(\varrho_{\epsilon}\right)-H_{\epsilon}\left(\widetilde{\varrho}_{\epsilon}\right)-H^{\prime}\left(\widetilde{\varrho}_{\epsilon}\right)\left(\varrho_{\epsilon}-\widetilde{\varrho}_{\epsilon}\right)\right]_{\mathrm{ess}}\right\|_{L^{1}(\Omega)} \\
& \leq \epsilon^{2} C \text {, } \\
& \text { ess } \sup _{t \in(0, T)}\left\|\left[H_{\epsilon}\left(\varrho_{\epsilon}\right)-H_{\epsilon}\left(\widetilde{\varrho}_{\epsilon}\right)-H^{\prime}\left(\widetilde{\varrho}_{\epsilon}\right)\left(\varrho_{\epsilon}-\widetilde{\varrho}_{\epsilon}\right)\right]_{\mathrm{res}}\right\|_{L^{1}(\Omega)} \\
& \leq \epsilon^{2} C \text {. }
\end{aligned}
$$

Using the estimate of (49) implies

$$
\text { ess } \sup _{t \in(0, T)}\left\|\left[\frac{\varrho_{\epsilon}-\widetilde{\varrho}_{\epsilon}}{\epsilon}\right]_{\mathrm{ess}}\right\|_{L^{2}(\Omega)} \leq C .
$$

Note that the static equation (29) holds

$$
\left\|\widetilde{\varrho}_{\epsilon}-\widetilde{\varrho}\right\|_{C(\bar{\Omega})} \leq \epsilon^{\alpha} C, \quad \widetilde{\varrho}_{\epsilon}=\widetilde{\varrho}=\varrho_{\infty} \quad \text { in } \Omega-\operatorname{supp}[F] .
$$

Due to the estimate of (51) and the convergence of (52), it follows that

$$
\text { ess } \sup _{t \in(0, T)}\left\|\left[\frac{\varrho_{\epsilon}-\tilde{\varrho}}{\epsilon}\right]_{\mathrm{ess}}\right\|_{L^{2}(\Omega)} \leq C .
$$

In order that we now derive the estimate of the velocity, we first verify that

$$
\text { ess } \sup _{t \in(0, T)}\left\|\left[\frac{\varrho_{\epsilon}-\tilde{\varrho}}{\epsilon}\right]_{\mathrm{res}}\right\|_{L^{2}(\Omega)} \leq C .
$$


To do this, we use the following inequality due to two assumptions of pressure (7) and (12),

$$
\frac{\partial^{2} H_{\epsilon}(\varrho)}{\partial \varrho^{2}}=\frac{p^{\prime}\left(\epsilon^{\alpha} \varrho\right)}{\varrho} \geq C\left(\frac{1}{\varrho}+\frac{\epsilon^{2 \alpha / 3}}{\varrho^{1 / 3}}\right),
$$

which deduce, thanks to (49),

$$
\begin{gathered}
\text { ess } \sup _{t \in(0, T)}[1]_{\mathrm{res}} \leq \epsilon^{2} C, \\
\text { ess } \sup _{t \in(0, T)}\left\|\left[\varrho_{\epsilon} \log \left(\varrho_{\epsilon}\right)\right]_{\mathrm{res}}\right\|_{L^{1}(\Omega)} \leq C \epsilon^{2}, \\
\text { ess } \sup _{t \in(0, T)}\left\|\left[\varrho_{\epsilon}\right]_{\mathrm{res}}\right\|_{L^{5 / 3}(\Omega)} \leq C \epsilon^{(6-2 \alpha) / 5} .
\end{gathered}
$$

Finally, we get

$$
\text { ess } \sup _{t \in(0, T)}\left\|\frac{\left[\varrho_{\epsilon}\right]_{\text {res }}}{\epsilon}\right\|_{L^{6 / 5}(\Omega)} \leq C \epsilon^{1 / 6}
$$

where we have used the above two estimates (57) and (58) and so the estimate (56) together with (59) shows (54).

We now derive the estimate of the velocity. Indeed, it is easy to show with a simple computation that

$$
\begin{gathered}
\operatorname{ess} \sup _{t \in(0, T)}\left\|\left[\mathbf{u}_{\epsilon}\right]_{\mathrm{ess}}\right\|_{L^{2}(\Omega)} \leq C \sup _{t>0} \int_{\Omega} \varrho_{\epsilon}\left|\mathbf{u}_{\epsilon}\right|^{2} \mathrm{~d} x \leq C, \\
\left\|\left[\mathbf{u}_{\epsilon}\right]_{\mathrm{res}}\right\|_{L^{2}(\Omega)}^{2} \leq C \int_{\Omega}\left|\varrho_{\epsilon}-\tilde{\varrho}\right|\left|\mathbf{u}_{\epsilon}\right|^{2} \mathrm{~d} x \leq \epsilon C\left\|\mathbf{u}_{\epsilon}\right\|_{L^{p}(\Omega)}^{2} \\
\leq \epsilon C\left\|\nabla \mathbf{u}_{\epsilon}\right\|_{L^{2}(\Omega)}^{2},
\end{gathered}
$$

for a certain $p>1$ where we have used the Sobolev embedding inequality. Thus we get

$$
\left\|\mathbf{u}_{\epsilon}\right\|_{L^{2}((0, T) \times \Omega)} \leq C
$$

and the estimates in (48) and (61) imply

$$
\left\|\mathbf{u}_{\epsilon}\right\|_{L^{2}\left(0, T ; H^{1}\left(\Omega ; \mathbb{R}^{3}\right)\right)} \leq C .
$$

4.2. Convergence of Anelastic Constraint. We will use the uniform estimate (62) to deduce

$$
\mathbf{u}_{\epsilon} \longrightarrow \mathbf{U} \quad \text { weakly in } L^{2}\left(0, T ; W^{1,2}\left(\Omega ; \mathbb{R}^{3}\right)\right)
$$

up to a subsequence of $\{\epsilon>0\}$. In accordance with (53) and (54), we obtain

$$
\varrho_{\epsilon} \longrightarrow \widetilde{\varrho} \text { in } L^{\infty}\left(0, T ; L^{p}(\Omega)\right), \text { for a certain } p>0,
$$

and so we can take the limit of $\epsilon$ in the continuity equation (20) to get

$$
\int_{0}^{T} \int_{\Omega} \widetilde{\varrho} \cdot \nabla_{x} \varphi \mathrm{d} x \mathrm{~d} t=0
$$

for all $\varphi \in C_{c}^{\infty}((0, T) \times \Omega)$.
4.3. Convergence of Moment Equation. To begin with, using two estimates (63) and (64),

$$
\begin{array}{r}
\varrho_{\epsilon} \mathbf{u}_{\epsilon} \longrightarrow \widetilde{\varrho} \mathbf{U} \text { weakly }-(*) \text { in } L^{\infty}\left(0, T ; L^{p}\left(\Omega ; \mathbb{R}^{3}\right)\right), \\
\text { for a certain } p>0 .
\end{array}
$$

Hence

$$
\varrho_{\epsilon} \mathbf{u}_{\epsilon} \otimes \mathbf{u}_{\epsilon} \longrightarrow \overline{\widetilde{\varrho} \otimes \mathbf{U}} \quad \text { weakly in } L^{\infty}\left(0, T ; L^{q}\left(\Omega ; \mathbb{R}^{3}\right)\right),
$$

for a certain $q>1$. Actually, we do not know $\overline{\varrho \mathbf{U} \otimes \mathbf{U}}=$ $\tilde{\varrho} \mathbf{U} \otimes \mathbf{U}$ due to the oscillations of the gradient component of the velocity field and we postpone this part to handle the oscillations of the gradient component in the next section.

We also need to get the uniform estimates for the directional field $\left\{\mathbf{d}_{\epsilon}\right\}_{\epsilon>0}$. Multiplying $\mathbf{d}_{\epsilon}$ on (27) yields

$$
\partial_{t}\left|\mathbf{d}_{\epsilon}\right|^{2}-\Delta\left|\mathbf{d}_{\epsilon}\right|^{2}+\mathbf{u}_{\epsilon} \cdot \nabla\left|\mathbf{d}_{\epsilon}\right|^{2} \leq 0,
$$

and so applying the maximum principle for weak solutions provides

$$
\left\{\mathbf{d}_{\epsilon}\right\}_{\epsilon>0} \text { bounded in } L^{\infty}([0, T] \times \Omega),
$$

which implies

$$
\left\{\nabla \mathbf{d}_{\epsilon}\right\}_{\epsilon>0} \text { bounded in } L^{2}\left([0, T] ; H^{2}(\Omega)\right),
$$

where we have used (47), the basic elliptic theory, and the following Gagliardo-Nirenberg inequality:

$$
\left\|\nabla \mathbf{d}_{\epsilon}\right\|_{L^{4}(\Omega)} \leq C\left\|\Delta \mathbf{d}_{\epsilon}\right\|_{L^{2}(\Omega)}^{1 / 2}\left\|\mathbf{d}_{\epsilon}\right\|_{L^{\infty}(\Omega)}^{1 / 2}+C\left\|\mathbf{d}_{\epsilon}\right\|_{L^{\infty}(\Omega)} .
$$

and thus we derive

$$
\left\{\partial_{t} \mathbf{d}_{\epsilon}\right\}_{\epsilon>0} \text { bounded in } L^{p}\left(0, T ; W^{-1,2}\left(\Omega ; \mathbb{R}^{3}\right)\right),
$$

for a certain $p>1$. Applying the Aubin-Lions lemma applied to (27) together with (47), (70), (72), and (61) implies

$$
\mathbf{d}_{\epsilon} \longrightarrow \mathbf{d} \text { strongly } L^{2}\left(0, T ; L^{2}\left(\Omega ; H^{1}(\Omega)\right)\right) \text {. }
$$

Thus we get the boundary condition $\left.\mathbf{d}\right|_{\partial \Omega}=\mathbf{d}_{0}$ and

$$
\begin{aligned}
\nabla \mathbf{d}_{\epsilon} & \otimes \nabla \mathbf{d}_{\epsilon}-\left(\frac{1}{2}\left|\nabla \mathbf{d}_{\epsilon}\right|^{2}+F\left(\mathbf{d}_{\epsilon}\right)\right) \square \\
& \longrightarrow \nabla \mathbf{d} \otimes \nabla \mathbf{d}-\left(\frac{1}{2}|\nabla \mathbf{d}|^{2}+F(\mathbf{d})\right)
\end{aligned}
$$

in the sense of distribution.

We are now able to identify the limit problem of the moment equation (22). To do this, we first rewrite the moment equation as follows:

$$
\begin{array}{r}
\partial_{t}\left(\varrho_{\epsilon} \mathbf{u}_{\epsilon}\right)+\operatorname{div}_{x}\left(\varrho_{\epsilon} \mathbf{u}_{\epsilon} \otimes \mathbf{u}_{\epsilon}\right)+\frac{1}{\epsilon^{2}} \nabla_{x}\left(p_{\epsilon}\left(\varrho_{\epsilon}\right)-p_{0} \widetilde{\varrho}_{\epsilon}\right) \\
+p_{0} \nabla\left(\frac{\varrho_{\epsilon}-\widetilde{\varrho}}{\epsilon^{2}}\right)+\left(\frac{\tilde{\varrho}-\varrho_{\epsilon}}{\epsilon^{2}}\right) \\
=\mu_{\epsilon} \Delta \mathbf{u}_{\epsilon}-v_{\epsilon} \operatorname{div}_{x}\left(\nabla \mathbf{d}_{\epsilon} \otimes \nabla \mathbf{d}_{\epsilon}\right. \\
\left.-\left(\frac{1}{2}\left|\nabla \mathbf{d}_{\epsilon}\right|^{2}+F\left(\mathbf{d}_{\epsilon}\right)\right) \mathbb{\square}_{3}\right),
\end{array}
$$


From the previous estimates, we get

$$
\begin{aligned}
\int_{0}^{T} \int_{\Omega} & \left(\widetilde{\varrho} \mathbf{U} \cdot \partial_{t} \varphi+\overline{\widetilde{\varrho} \mathbf{U} \otimes \mathbf{U}}: \nabla_{x} \varphi\right) \mathrm{d} x \mathrm{~d} t \\
= & \int_{0}^{T} \int_{\Omega}\left(\mu \nabla_{x} \mathbf{U}: \nabla_{x} \varphi-v(\nabla \mathbf{d} \otimes \nabla \mathbf{d}): \nabla_{x} \varphi\right) \mathrm{d} x \mathrm{~d} t \\
& -\int_{\Omega}(\widetilde{\varrho} \mathbf{U})_{0} \cdot \varphi \mathrm{d} x
\end{aligned}
$$

for any test function

$$
\varphi \in C_{c}^{\infty}\left([0, T] \times \Omega ; \mathbb{R}^{3}\right), \quad \operatorname{div}_{x} \varphi=0,
$$

if we show

$$
\frac{1}{\epsilon^{2}} \int_{0}^{T} \int_{\Omega}\left(p_{\epsilon}\left(\varrho_{\epsilon}\right)-p_{0} \varrho_{\epsilon}\right) \mathrm{d} x \mathrm{~d} t \longrightarrow 0 \quad \text { as } \epsilon \longrightarrow 0,
$$

where we have here used (8), (53), and (54). It remains to show that

$$
\int_{0}^{T} \int_{\Omega} \overline{\widetilde{\varrho} \mathbf{U} \otimes \mathbf{U}}: \nabla_{x} \varphi \mathrm{d} x \mathrm{~d} t=\int_{0}^{T} \int_{\Omega}[\widetilde{\varrho} \mathbf{U} \otimes \mathbf{U}]: \nabla_{x} \varphi \mathrm{d} x \mathrm{~d} t
$$

for any

$$
\varphi \in C_{c}^{\infty}\left((0, T) \times \Omega ; \mathbb{R}^{3}\right), \quad \operatorname{div}_{x} \varphi=0 .
$$

The proofs of (78) and (79) are provided in the next two sections.

4.4. Pressure Estimate. The next challenge is to establish uniform bounds on the pressure as well as on the internal energy in terms of a reflexive space $L^{q}$, with $q>1$. We here use the Bogovski operator. Let us take a test function and adapt this test function in the moment equation (2):

$$
\begin{array}{r}
\varphi(t, x)=\psi(t) \mathscr{B}\left[b\left(\varrho_{\epsilon}\right)-\frac{1}{|\Omega|} \int_{\Omega} b\left(\varrho_{\epsilon}\right) \mathrm{d} x\right], \\
\psi \in \mathscr{D}(0, T) .
\end{array}
$$

We will write the moment equation (2) with simple computations again:

$$
\begin{aligned}
\frac{1}{\epsilon^{2}} \int_{0}^{T} \int_{\Omega} \psi p_{\epsilon}\left(\varrho_{\epsilon}\right) b\left(\varrho_{\epsilon}\right) \mathrm{d} x \mathrm{~d} t \\
=\frac{1}{\epsilon^{2}|\Omega|} \int_{0}^{T} \int_{\Omega} \psi p_{\epsilon}\left(\varrho_{\epsilon}\right) \mathrm{d} x\left(\int_{\Omega} b\left(\varrho_{\epsilon}\right) \mathrm{d} x\right) \mathrm{d} t \\
\quad-\frac{1}{\epsilon^{2}} \int_{0}^{T} \int_{\Omega} \psi \varrho_{\epsilon} \nabla G \\
\cdot \mathscr{B}\left[b\left(\varrho_{\epsilon}\right)-\frac{1}{|\Omega|} \int_{\Omega} b\left(\varrho_{\epsilon}\right) \mathrm{d} x\right] \mathrm{d} x \mathrm{~d} t \\
-\int_{0}^{T} \int_{\Omega} \psi \Pi_{\epsilon} \\
\quad \mathscr{B}\left[b\left(\varrho_{\epsilon}\right)-\frac{1}{|\Omega|} \int_{\Omega} b\left(\varrho_{\epsilon}\right) \mathrm{d} x\right] \mathrm{d} x \mathrm{~d} t+J_{\epsilon},
\end{aligned}
$$

where

$$
\Pi_{\epsilon}:=-v_{\epsilon} \operatorname{div}_{x}\left(\nabla \mathbf{d}_{\epsilon} \otimes \nabla \mathbf{d}_{\epsilon}-\left(\frac{1}{2}\left|\nabla \mathbf{d}_{\epsilon}\right|^{2}+F\left(\mathbf{d}_{\epsilon}\right)\right) \rrbracket_{3}\right),
$$

and $J_{\epsilon}$ is defined by

$$
\begin{aligned}
J_{\epsilon}= & \int_{0}^{T} \int_{\Omega} \psi \mu_{\epsilon} \nabla_{x} \mathbf{u}_{\epsilon}: \nabla \mathscr{B}\left[b\left(\varrho_{\epsilon}\right)-\frac{1}{|\Omega|} \int_{\Omega} b\left(\varrho_{\epsilon}\right) \mathrm{d} x\right] \mathrm{d} x \mathrm{~d} t \\
& -\int_{0}^{T} \int_{\Omega} \psi \varrho_{\epsilon} \mathbf{u}_{\epsilon} \otimes \mathbf{u}_{\epsilon} \\
& : \nabla \mathscr{B}\left[b\left(\varrho_{\epsilon}\right)-\frac{1}{|\Omega|} \int_{\Omega} b\left(\varrho_{\epsilon}\right) \mathrm{d} x\right] \mathrm{d} x \mathrm{~d} t \\
& -\int_{0}^{T} \int_{\Omega} \partial_{t} \psi \varrho_{\epsilon} \mathbf{u}_{\epsilon} \cdot \mathscr{B}\left[b\left(\varrho_{\epsilon}\right)-\frac{1}{|\Omega|} \int_{\Omega} b\left(\varrho_{\epsilon}\right) \mathrm{d} x\right] \mathrm{d} x \mathrm{~d} t \\
& +\int_{0}^{T} \int_{\Omega} \psi \varrho_{\epsilon} \mathbf{u}_{\epsilon} \cdot \mathscr{B}\left[\operatorname{div}_{x}\left(b\left(\varrho_{\epsilon}\right) \mathbf{u}_{\epsilon}\right)\right] \mathrm{d} x \mathrm{~d} t \\
& +\int_{0}^{T} \psi \int_{\Omega} \varrho_{\epsilon} \mathbf{u}_{\epsilon} \\
& \cdot \mathscr{B}\left[\left(\varrho_{\epsilon} b^{\prime}\left(\varrho_{\epsilon}\right)-b\left(\varrho_{\epsilon}\right)\right) \operatorname{div}_{x} \mathbf{u}_{\epsilon}\right. \\
& \left.-\frac{1}{|\Omega|} \int_{\Omega}\left(b\left(\varrho_{\epsilon}\right)-b^{\prime}\left(\varrho_{\epsilon}\right) \varrho_{\epsilon}\right) \operatorname{div} \mathbf{u}_{\epsilon} \mathrm{d} x\right] \mathrm{d} x \mathrm{~d} t .
\end{aligned}
$$

We notice that all estimates in (84) are uniformly bounded due to the uniform estimates in the previous section if we take a special $b$ verifying

$$
|b(\varrho)|+\left|\varrho b^{\prime}(\varrho)\right| \leq C \varrho^{\gamma}
$$

with a certain $\gamma \in(0,1)$. In virtue of $(57)$, it is easy to see

$$
\text { ess } \sup _{t \in(0, T)} \int_{\Omega} b\left(\varrho_{\epsilon}\right) \mathrm{d} x \leq C \epsilon^{2}
$$

and so we get

$$
\left\|b\left(\varrho_{\epsilon}\right)\right\|_{L^{q}(\Omega)}^{q} \leq\left\|\left[\varrho_{\epsilon}\right]_{\mathrm{res}}^{\gamma q}\right\|_{L^{1}(\Omega)} \leq\left\|\left[\varrho_{\epsilon} \log \varrho_{\epsilon}\right]_{\mathrm{res}}^{\gamma q}\right\|_{L^{1}(\Omega)} \leq C \epsilon^{2}
$$

for $\gamma<1 / q$ and the first integral of the right hand side of (82) is uniformly bounded. We now need to control the first and second integrals of the right hand side of (82). To do this, let 
us rewrite the first term into the following form with using the static problem (8)

$$
\begin{aligned}
\frac{1}{\epsilon^{2}} \int_{\Omega} \varrho_{\epsilon} \nabla G \cdot \mathscr{B}\left[b\left(\varrho_{\epsilon}\right)-\frac{1}{|\Omega|} \int_{\Omega} b\left(\varrho_{\epsilon}\right) \mathrm{d} x\right] \mathrm{d} x \\
=\frac{1}{\epsilon} \int_{\Omega}\left[\frac{\varrho_{\epsilon}-\tilde{\varrho}}{\epsilon}\right]_{\mathrm{ess}} \nabla G \\
\cdot \mathscr{B}\left[b\left(\varrho_{\epsilon}\right)-\frac{1}{|\Omega|} \int_{\Omega} b\left(\varrho_{\epsilon}\right) \mathrm{d} x\right] \mathrm{d} x \\
+\frac{1}{\epsilon} \int_{\Omega}\left[\frac{\varrho_{\epsilon}-\tilde{\varrho}}{\epsilon}\right]_{\mathrm{res}} \nabla G \\
\cdot \mathscr{B}\left[b\left(\varrho_{\epsilon}\right)-\frac{1}{|\Omega|} \int_{\Omega} b\left(\varrho_{\epsilon}\right) \mathrm{d} x\right] \mathrm{d} x \\
-\frac{p_{0}}{\epsilon^{2}} \int_{\Omega} \tilde{\varrho}\left[b\left(\varrho_{\epsilon}\right)-\frac{1}{|\Omega|} \int_{\Omega} b\left(\varrho_{\epsilon}\right) \mathrm{d} x\right] \mathrm{d} x
\end{aligned}
$$

where the last integral is uniformly bounded due to (86). Following estimates (53), (56), and (87) together with the $L^{p_{-}}$ estimates for $\mathscr{B}$, one gets the uniform boundedness of the first and second terms of the right hand side of (88).

On the other hand, the estimates in (86) and (87) together with the $L^{p}$-estimates for $\mathscr{B}$ yield that the third term of the right hand side of (82) is uniformly bounded. Consequently, we deduce that

$$
\int_{0}^{T} \int_{\Omega}\left[p_{\epsilon}\left(\varrho_{\epsilon}\right)\right]_{\mathrm{res}} \varrho_{\epsilon}^{v} \mathrm{~d} x \mathrm{~d} t \leq C \epsilon^{2},
$$

for a certain $v \in(0,1)$. We first split the integration of (78) into two parts to show (78):

$$
\begin{aligned}
\frac{1}{\epsilon^{2}} \int_{0}^{T} \int_{\Omega}\left|p_{0} \varrho_{\epsilon}-p_{\epsilon}\left(\varrho_{\epsilon}\right)\right| \mathrm{d} x \mathrm{~d} t \\
=\frac{1}{\epsilon^{2}} \int_{0}^{T} \int_{\varrho_{\epsilon} \leq M}\left|p_{0} \varrho_{\epsilon}-p_{\epsilon}\left(\varrho_{\epsilon}\right)\right| \mathrm{d} x \mathrm{~d} t \\
\quad+\frac{1}{\epsilon^{2}} \int_{0}^{T} \int_{\varrho_{\epsilon}>M}\left|p_{0} \varrho_{\epsilon}-p_{\epsilon}\left(\varrho_{\epsilon}\right)\right| \mathrm{d} x \mathrm{~d} t .
\end{aligned}
$$

For the first one of the right hand side, we get

$$
\begin{array}{r}
\frac{1}{\epsilon^{2}} \int_{0}^{T} \int_{\varrho_{\epsilon} \leq M}\left|p_{0} \varrho_{\epsilon}-p_{\epsilon}\left(\varrho_{\epsilon}\right)\right| \mathrm{d} x \mathrm{~d} t \\
\quad \leq \frac{\epsilon^{\alpha-2} M^{2}}{2} \sup _{0 \leq r \leq M} P^{\prime \prime}(r)|\Omega|,
\end{array}
$$

where we have used the Taylor expansion of degree 2. We also use (57) and (89) to prove the second part of the right hand side.

4.5. Convergence of the Convective Term. In this section, our aim is to show that (79) holds. Before we prove (79), we will introduce the Helmholtz decomposition and the following material may be found in most of the text book of fluid mechanics. Let us denote $L_{1 / \widetilde{\varrho}}^{2}$ by the Hilbert space with the inner product

$$
\langle\mathbf{v}, \mathbf{w}\rangle_{1 / \tilde{\varrho}}=\int_{\Omega} \mathbf{v} \cdot \mathbf{w} \frac{\mathrm{d} x}{\widetilde{\varrho}}
$$

and $D^{1,2}$ by the completion of $C_{c}^{\infty}(\bar{\Omega})$ with respect to the norm $\left\|\nabla_{x} \varphi\right\|_{L^{2}}(\Omega)$.

Theorem 4. For $\mathbf{v} \in L_{1 / \widetilde{\varrho}}^{2}\left(\Omega: \mathbb{R}^{3}\right)$, a vector function $\mathbf{v}: \Omega \rightarrow$ $\mathbb{R}^{3}$ is written as

$$
\mathbf{v}=\mathbf{P}_{\widetilde{\varrho}}[\mathbf{v}]+\mathbf{Q}_{\widetilde{\varrho}}[\mathbf{v}],
$$

where

$$
\mathbf{Q}_{\widetilde{\varrho}}[\mathbf{v}]=\widetilde{\varrho} \nabla_{x} \Psi
$$

and $\Psi$ is uniquely determined as the following Neumann problem:

$$
\begin{gathered}
\operatorname{div}_{x}\left(\widetilde{\varrho} \nabla_{x} \Psi\right)=\operatorname{div}_{x} \mathbf{v} \quad \text { in } \Omega, \\
\left.\widetilde{\varrho} \nabla_{x} \Psi \cdot \mathbf{n}\right|_{\partial \Omega}=0, \quad \int_{\Omega} \Psi d x=0,
\end{gathered}
$$

where $\mathbf{n}$ is the outward unit normal to $\partial \Omega$.

We now write

$$
\begin{aligned}
\varrho_{\epsilon} \mathbf{u}_{\epsilon} \otimes \mathbf{u}_{\epsilon}= & \mathbf{P}_{\widetilde{\varrho}}\left[\varrho_{\epsilon} \mathbf{u}_{\epsilon}\right] \otimes \mathbf{u}_{\epsilon}+\mathbf{Q}_{\widetilde{\varrho}}\left[\varrho_{\epsilon} \mathbf{u}_{\epsilon}\right] \otimes \mathbf{P}_{\widetilde{\varrho}}\left[\mathbf{u}_{\epsilon}\right] \\
& +\mathbf{Q}_{\widetilde{\varrho}}\left[\varrho_{\epsilon} \mathbf{u}_{\epsilon}\right] \otimes \mathbf{Q}_{\widetilde{\varrho}}\left[\mathbf{u}_{\epsilon}\right] .
\end{aligned}
$$

Let us first show

$$
\mathbf{P}_{\widetilde{\varrho}}\left[\varrho_{\epsilon} \mathbf{u}_{\epsilon}\right] \longrightarrow \mathbf{P}_{\widetilde{\varrho}}[\widetilde{\varrho} \mathbf{U}]=\widetilde{\varrho} \mathbf{U} \quad \text { in } L^{1}\left((0, T) \times \Omega ; \mathbb{R}^{3}\right)
$$

To do this, we adapt the following test function to the moment equation (22):

$$
\begin{gathered}
\varphi(t, x)=\frac{\varphi(t)}{\widetilde{\varrho}} \mathbf{P}_{\widetilde{\varrho}}[\widetilde{\varrho} \Psi], \quad \Psi \in C_{c}^{\infty}\left(\bar{\Omega} ; \mathbb{R}^{3}\right), \\
\left.\Psi \cdot \mathbf{n}\right|_{\partial \Omega}=0, \quad \psi \in C_{c}^{\infty}(0, T) .
\end{gathered}
$$

Taking into account the uniform estimates obtained in the previous sections, it follows that

$$
t \in[0, T] \longmapsto \int_{\Omega} \mathbf{P}_{\widetilde{\varrho}}\left[\varrho_{\epsilon} \mathbf{u}_{\epsilon}\right] \cdot \Psi \mathrm{d} x
$$

precompact in $C[0, T]$ and so the uniform estimates (45) and (64) with using the Sobolev embedding $L^{5 / 4}(\Omega) \hookrightarrow$ $\left[W^{1,2}(\Omega)\right]^{*}$ imply that

$$
\begin{gathered}
\mathbf{P}_{\widetilde{\varrho}}\left[\varrho_{\epsilon} \mathbf{u}_{\epsilon}\right] \longrightarrow \mathbf{P}_{\widetilde{\varrho}}[\widetilde{\varrho} \mathbf{U}]=\widetilde{\varrho} \mathbf{U} \\
\operatorname{in} C_{\text {weak }}\left([0, T] ; L^{5 / 4}\left(\Omega ; \mathbb{R}^{3}\right)\right) .
\end{gathered}
$$


Notice that

$$
\begin{array}{r}
\int_{0}^{T} \int_{\Omega} \mathbf{P}_{\widetilde{\varrho}}\left[\varrho_{\epsilon} \mathbf{u}_{\epsilon}\right] \cdot \mathbf{P}_{\widetilde{\varrho}}\left[\widetilde{\varrho} \mathbf{u}_{\epsilon}\right] \frac{\mathrm{d} x}{\widetilde{\varrho}} \mathrm{d} t \\
\quad=\int_{0}^{T} \int_{\Omega} \mathbf{P}_{\widetilde{\varrho}}\left[\varrho_{\epsilon} \mathbf{u}_{\epsilon}\right] \cdot \mathbf{u}_{\epsilon} \mathrm{d} x \mathrm{~d} t \\
\longrightarrow \int_{0}^{T} \int_{\Omega} \mathbf{P}_{\widetilde{\varrho}}[\widetilde{\varrho} \mathbf{U}] \cdot \mathbf{U} \mathrm{d} x \mathrm{~d} t \\
=\int_{0}^{T} \int_{\Omega} \widetilde{\varrho}^{2}|\mathbf{U}|^{2} \frac{\mathrm{d} x}{\widetilde{\varrho}} \mathrm{d} t,
\end{array}
$$

where we have here used (100). In virtue of (64) and (101), one gets

$$
\mathbf{P}_{\widetilde{\varrho}}\left[\varrho_{\epsilon} \mathbf{u}_{\epsilon}\right] \longrightarrow \widetilde{\varrho} \mathbf{U} \quad \text { in } L^{2}\left((0, T) \times \Omega ; \mathbb{R}^{3}\right)
$$

Since the Helmholtz projections

$$
\mathbf{v} \longrightarrow \mathbf{P}_{\widetilde{\varrho}}[\mathbf{v}], \quad \mathbf{v} \longrightarrow \mathbf{Q}_{\widetilde{\varrho}}[\mathbf{v}]
$$

map continuously the spaces $L^{p}\left(\Omega ; \mathbb{R}^{3}\right)$ and $W^{1, p}\left(\Omega ; \mathbb{R}^{3}\right)$ into itself for any $1<p$, it is easily seen that

$$
\mathbf{Q}_{\tilde{\varrho}}\left[\varrho_{\epsilon} \mathbf{u}_{\epsilon}\right] \longrightarrow 0 \quad \text { weakly }-(*) \text { in } L^{\infty}\left(0, T ; L^{5 / 4}\left(\Omega ; \mathbb{R}^{3}\right)\right) \text {. }
$$

In order to show (79), we should prove

$$
\lim _{\epsilon \rightarrow 0} \int_{0}^{T} \int_{\Omega} \mathbf{Q}_{\widetilde{\varrho}}\left[\varrho_{\epsilon} \mathbf{u}_{\epsilon}\right] \otimes \mathbf{Q}_{\widetilde{\varrho}}\left[\varrho_{\epsilon} \mathbf{u}_{\epsilon}\right]: \nabla_{x}\left(\frac{\varphi}{\widetilde{\varrho}}\right) \frac{\mathrm{d} x}{\widetilde{\varrho}} \mathrm{d} t=0,
$$

for any

$$
\varphi \in C_{c}^{\infty}\left((0, T) \times \bar{\Omega} ; \mathbb{R}^{3}\right), \quad \operatorname{div}_{x} \varphi=0,\left.\quad \varphi \cdot \mathbf{n}\right|_{\partial \Omega}=0 .
$$

We next study the acoustic equations. The acoustic equations are used to describe the time evolution of fast acoustic waves in the compressible models in order to handle the oscillation of $\mathbf{Q}\left[\varrho_{\epsilon} \mathbf{u}_{\epsilon}\right]$. To begin with, we rewrite (1) and (2):

$$
\begin{gathered}
\epsilon \partial_{t} X_{\epsilon}+\frac{1}{\widetilde{\varrho}} \operatorname{div}_{x} \mathbf{V}_{\epsilon}=0 \\
\epsilon \partial_{t} \mathbf{V}_{\epsilon}+p_{0} \widetilde{\varrho} \nabla_{x} X_{\epsilon}=\epsilon\left(\operatorname{div}_{x} \mathbf{G}_{\epsilon}^{1}+\nabla \mathbf{G}_{\epsilon}^{2}\right),
\end{gathered}
$$

where the previous estimates provide

$$
\begin{aligned}
& \mathbf{G}_{\epsilon}^{1} \text { is bounded in } L^{p}((0, T) \times \Omega)^{3 \times 3} \\
& \mathbf{G}_{\epsilon}^{2} \text { is bounded in } L^{p}((0, T) \times \Omega)^{3},
\end{aligned}
$$

for a certain $p>1$ and

$$
\begin{gathered}
X_{\epsilon}=\frac{\varrho_{\epsilon}-\widetilde{\varrho}}{\epsilon \widetilde{\varrho}}, \quad \mathbf{V}_{\epsilon}=\varrho_{\epsilon} \mathbf{u}_{\epsilon} \\
\mathbf{G}_{\epsilon}^{1}=\mu_{\epsilon} \nabla \mathbf{u}_{\epsilon}-\left(\varrho_{\epsilon} \mathbf{u}_{\epsilon} \otimes \mathbf{u}_{\epsilon}\right)-\nu_{\epsilon}\left(\nabla \mathbf{d}_{\epsilon} \otimes \nabla \mathbf{d}_{\epsilon}\right) \\
\mathbf{G}_{\epsilon}^{2}=\frac{1}{2}\left|\nabla \mathbf{d}_{\epsilon}\right|^{2}+F\left(\mathbf{d}_{\epsilon}\right) \\
-\left(H_{\epsilon}\left(\varrho_{\epsilon}\right)-H_{\epsilon}\left(\widetilde{\varrho}_{\epsilon}\right)-H^{\prime}\left(\widetilde{\varrho}_{\epsilon}\right)\left(\varrho_{\epsilon}-\widetilde{\varrho}_{\epsilon}\right)\right) .
\end{gathered}
$$

We now use the method of spectral analysis of the wave operator. Let us consider the eigenvalue problem:

$$
\widetilde{\varrho} \nabla_{x}\left(\frac{\mathbf{A}}{\widetilde{\varrho}}\right)=\lambda \mathbf{W}, \quad p_{0} \operatorname{div}_{x} \mathbf{W}=\lambda \mathbf{A}, \quad \text { in } \Omega,
$$

with the boundary condition

$$
\left.\mathbf{W} \cdot \mathbf{n}\right|_{\partial \Omega}=0 .
$$

Thus, the eigenvalue problem (112) and (113) can be written into the following form:

$$
-\operatorname{div}_{x}\left[\widetilde{\varrho} \nabla_{x}\left(\frac{\mathbf{A}}{\widetilde{\varrho}}\right)\right]=\Lambda \widetilde{\varrho}\left(\frac{\mathbf{A}}{\widetilde{\varrho}}\right), \quad \text { in } \Omega,
$$

with

$$
\left.\nabla_{x}\left(\frac{\mathbf{A}}{\tilde{\varrho}}\right) \cdot \mathbf{n}\right|_{\partial \Omega}=0, \quad \lambda^{2}=-\Lambda p_{0} .
$$

Following the eigenvalue problem (114) and (115), it is well known that there is an orthonormal basis $\left\{\mathbf{A}_{j, m}\right\}_{j=0, m=1}^{\infty, m_{j}}$ of real eigenfunctions of the weighed Lebesgue space $L_{1 / \widetilde{\varrho}}^{2}(\Omega)$ corresponding to eigenvalues $\Lambda_{j, m}$ such that

$$
\begin{gathered}
m_{0}=1, \quad \Lambda_{0,1}, \quad \mathbf{A}_{0,1}=\tilde{\varrho} \\
0<\Lambda_{1,1}=\cdots=\Lambda_{0, m_{1}}\left(=\Lambda_{1}\right)<\Lambda_{2,1} \\
=\cdots=\Lambda_{2, m_{2}}\left(=\Lambda_{2}\right)<\cdots,
\end{gathered}
$$

where $m_{j}$ stands for the multiplicity of $\Lambda_{j}$. We also see that $\left\{\mathbf{W}_{j, m}\right\}_{j=0, m=1}^{\infty, m_{j}}$ is an orthonormal basis in $\mathbf{Q}\left[L_{1 / \widetilde{\varrho}}^{2}(\Omega)^{3}\right]$ defined by

$$
\begin{aligned}
& \mathbf{W}_{ \pm j, m}=\frac{\mp i}{\sqrt{p_{0} \Lambda_{j}}} \widetilde{\varrho} \nabla_{x} \frac{\mathbf{A}_{j, m}}{\widetilde{\varrho}}, \\
& j=1,2, \ldots, m, m=1, \ldots, m_{j} .
\end{aligned}
$$

We now take $\varphi=\psi_{1}(t) \mathbf{A}_{j, m}$ and $\phi=\psi_{2}(t) \mathbf{W}_{ \pm j, m}$ as test functions to the system (107), and then we obtain

$$
\begin{aligned}
& \epsilon \partial_{t}\left[X_{\epsilon}\right]_{j, m}-p_{0} \sqrt{\Lambda_{j}} \operatorname{div}_{x}\left[\mathbf{V}_{\epsilon}\right]_{j, m}=0, \\
& \epsilon \partial_{t}\left[\mathbf{V}_{\epsilon}\right]_{j, m}+\sqrt{\Lambda_{j}}\left[X_{\epsilon}\right]_{j, m}=\epsilon\left[B_{\epsilon}\right]_{j, m},
\end{aligned}
$$


for $j=1,2, \ldots$, and $m=1, \ldots, m_{j}$, where $\left[X_{\epsilon}\right]_{j, m},\left[\mathbf{V}_{\epsilon}\right]_{j, m}$ are defined by

$$
\begin{gathered}
{\left[X_{\epsilon}\right]_{j, m}=\int_{\Omega} X_{\epsilon} \mathbf{A}_{j, m} \mathrm{~d} x} \\
{\left[\mathbf{V}_{\epsilon}\right]_{j, m}=\frac{i}{\sqrt{p_{0}}} \int_{\Omega} \mathbf{V}_{\epsilon} \cdot \mathbf{W}_{j, m} \mathrm{~d} x}
\end{gathered}
$$

and the estimates in (108) show that

$$
\left\{\left[B_{\epsilon}\right]_{j, m}\right\}_{\epsilon>0} \text { is bounded in } L^{1}(0, T) \text { for any fix } j, m \text {. }
$$

Moreover, in virtue of section 5 in [7] for a finite number of modes, we set

$$
\mathbf{Q}_{\tilde{\varrho}, N}[\widetilde{\varrho} \mathbf{Z}]=\frac{-i}{\sqrt{p_{0}}} \sum_{j, 0<\Lambda_{j} \leq N} \sum_{m=1}^{m_{j}}[\widetilde{\varrho} \mathbf{Z}]_{j, m} \mathbf{W}_{j, m} .
$$

We first note

$$
\begin{aligned}
& \left\|\mathbf{Q}_{\tilde{\varrho}}\left[\widetilde{\varrho} \mathbf{u}_{\epsilon}\right]-\mathbf{Q}_{\widetilde{\rho}, N}\left[\widetilde{\varrho} \mathbf{u}_{\epsilon}\right]\right\|_{L_{1 / \widetilde{\varrho}}^{2}\left(\Omega ; \mathbb{R}^{3}\right)}^{2} \\
& \quad \leq \frac{1}{N}\left\|\operatorname{div}_{x}\left(\widetilde{\varrho} \mathbf{u}_{\epsilon}\right)\right\|_{L_{1 / \widetilde{\varrho}}^{2}}^{2}(\Omega) \longrightarrow 0,
\end{aligned}
$$

as $N \rightarrow \infty$ in $L^{2}\left(0, T ; L_{1 / \tilde{\varrho}}^{2}\left(\Omega ; \mathbb{R}^{3}\right)\right)$ where we here have used the Parseval identity and (121) (see section 6.6 of [7]). Thus it is sufficient to show (105) for $\mathbf{Q}_{\widetilde{\varrho}, N}$ instead of $\mathbf{Q}_{\tilde{\rho}}$. Observe that, thanks to (114) and (121), it is easy to see

$$
\begin{gathered}
\Psi_{\epsilon}=\frac{1}{p_{0}} \sum_{j=1}^{N} \sum_{m=1}^{m_{j}} \frac{\left[\mathbf{V}_{\epsilon}\right]_{j, m}}{\sqrt{\Lambda_{j}}}\left(\frac{\mathbf{A}_{j, m}}{\widetilde{\varrho}}\right), \\
-\operatorname{div}_{x}\left(\widetilde{\varrho} \nabla_{x} \Psi_{\epsilon}\right)=\frac{1}{p_{0}} \sum_{j=1}^{N} \sum_{m=1}^{m_{j}} \sqrt{\Lambda_{j}}\left[\mathbf{V}_{\epsilon}\right]_{j, m} \mathbf{A}_{j, m} .
\end{gathered}
$$

We are now ready to show (105) and so we now use (118) and (123) to rewrite the oscillation part $\mathbf{Q}_{\tilde{\varrho}}\left[\varrho_{\epsilon} \mathbf{u}_{\epsilon}\right]$ as

$$
\begin{array}{r}
\lim _{\epsilon \rightarrow 0} \int_{0}^{T} \int_{\Omega}\left(\mathbf{Q}_{\tilde{\varrho}}\left[\varrho_{\epsilon} \mathbf{u}_{\epsilon}\right] \otimes \mathbf{Q}_{\tilde{\varrho}}\left[\varrho_{\epsilon} \mathbf{u}_{\epsilon}\right]\right): \nabla_{x}\left(\frac{\varphi}{\widetilde{\varrho}}\right) \mathrm{d} x \mathrm{~d} t \\
\quad=\lim _{\epsilon \rightarrow 0} \int_{0}^{T} \int_{\Omega}\left(\widetilde{\varrho} \nabla_{x} \Psi_{\epsilon} \otimes \nabla_{x} \Psi_{\epsilon}\right): \nabla_{x}\left(\frac{\varphi}{\widetilde{\varrho}}\right) \mathrm{d} x \mathrm{~d} t
\end{array}
$$

$$
\begin{aligned}
= & -\lim _{\epsilon \rightarrow 0} \int_{0}^{T} \int_{\Omega} \operatorname{div}_{x}\left(\widetilde{\varrho} \nabla_{x} \Psi_{\epsilon}\right) \nabla_{x} \Psi_{\epsilon}: \nabla_{x}\left(\frac{\varphi}{\widetilde{\varrho}}\right) \mathrm{d} x \mathrm{~d} t \\
= & -\frac{\epsilon}{p_{0}^{2}} \lim _{\epsilon \rightarrow 0} \int_{0}^{T} \int_{\Omega} \sum_{j=1}^{N} \sum_{m=1}^{m_{j}} \sqrt{\Lambda_{j}}\left[X_{\epsilon}\right]_{j, m} \\
& \times \frac{\left[\mathbf{A}_{j, m}\right]}{\widetilde{\varrho}} \nabla_{x} \Psi_{\epsilon} \cdot \partial_{t} \varphi \mathrm{d} x \mathrm{~d} t \\
& +\frac{\epsilon}{p_{0}^{2}} \lim _{\epsilon \rightarrow 0} \int_{0}^{T} \int_{\Omega} \sum_{j=1}^{N} \sum_{m=1}^{m_{j}} \sqrt{\Lambda_{j}}\left[X_{\epsilon}\right]_{j, m} \\
& \times \frac{\left[\mathbf{A}_{j, m}\right]}{\widetilde{\varrho}} \partial_{t} \nabla_{x} \Psi_{\epsilon} \cdot \varphi \mathrm{d} x \mathrm{~d} t
\end{aligned}
$$

which converges to 0 as $\epsilon$ tends to 0 where we can see this proof in section 6.6 of Feireisl and Novotný [7].

\section{Acknowledgment}

The work of Y.-S. Kwon was supported by Basic Science Research Program through the National Research Foundation of Korea (NRF) funded by the Ministry of Education, Science and Technology (2012-0003611).

\section{References}

[1] E. Feireisl, A. Novotný, and H. Petzeltová, “On the incompressible limit for the Navier-Stokes-Fourier system in domains with wavy bottoms," Mathematical Models \& Methods in Applied Sciences, vol. 18, no. 2, pp. 291-324, 2008.

[2] D. Wang and C. Yu, "Incompressible limit for the compressible flow of liquid crystals," http://arxiv.org/abs/1108.4941/.

[3] D. Wang and C. Yu, "Global weak solution and large-time behavior for the compressible flow of liquid crystals," Archive for Rational Mechanics and Analysis, vol. 204, no. 3, pp. 881-915, 2012.

[4] X.-G. Liu and J. Qing, "Globally weak solution to the flow of compressible liquid crystals system," Discrete and Continuous Dynamical Systems A, vol. 33, no. 2, pp. 757-788, 2013.

[5] S. Klainerman and A. Majda, "Singular limits of quasilinear hyperbolic systems with large parameters and the incompressible limit of compressible fluids," Communications on Pure and Applied Mathematics, vol. 34, no. 4, pp. 481-524, 1981.

[6] P.-L. Lions and N. Masmoudi, "Incompressible limit for a viscous compressible fluid," Journal de Mathématiques Pures et Appliquées, vol. 77, no. 6, pp. 585-627, 1998.

[7] E. Feireisl and A. Novotný, Singular Limits in Thermodynamics of Viscous Fluids, Advances in Mathematical Fluid Mechanics, Birkhäuser, Basel, Switzerland, 2009.

[8] E. Feireisl and A. Novotný, "The low mach number limit for the full Navier-Stokes-Fourier system," Archive for Rational Mechanics and Analysis, vol. 186, no. 1, pp. 77-107, 2007.

[9] Y.-S. Kwon and K. Trivisa, "On the incompressible limits for the full magnetohydrodynamics flows," Journal of Differential Equations, vol. 251, no. 7, pp. 1990-2023, 2011. 
[10] A. Novotný, M. Růocircužička, and G. Thäter, "Singular limit of the equations of magnetohydrodynamics in the presence of strong stratification," Mathematical Models and Methods in Applied Sciences, vol. 21, no. 1, pp. 115-147, 2011.

[11] G. Lee, P. Kim, and Y.-S. Kwon, "Incompressible limit for the full magnetohydrodynamics flows under strong stratification," Journal of Mathematical Analysis and Applications, vol. 387, no. 1, pp. 221-240, 2012.

[12] E. Feireisl, A. Novotný, and H. Petzeltová, "Low Mach number limit for the Navier-Stokes system on unbounded domains under strong stratification," Communications in Partial Differential Equations, vol. 35, no. 1, pp. 68-88, 2010. 


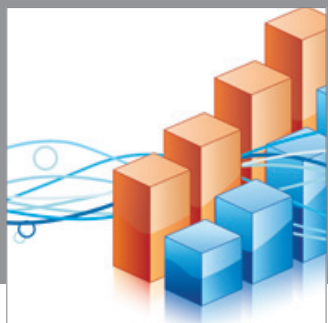

Advances in

Operations Research

mansans



The Scientific World Journal
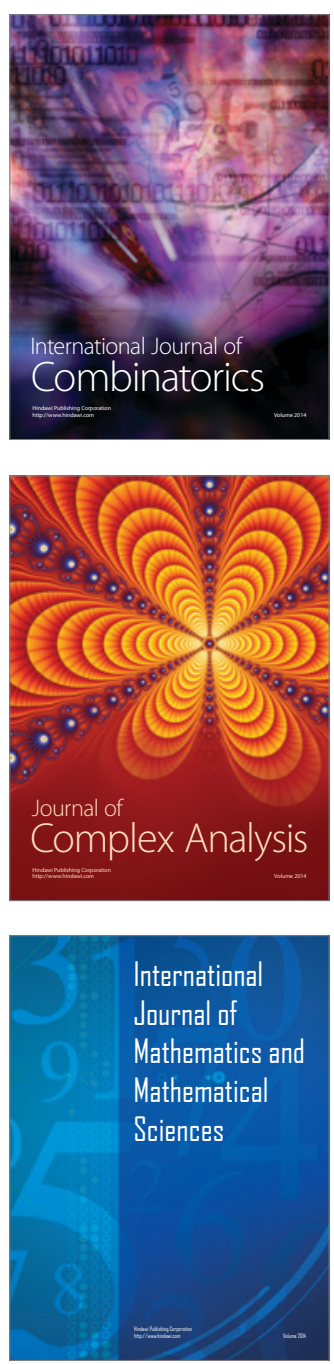
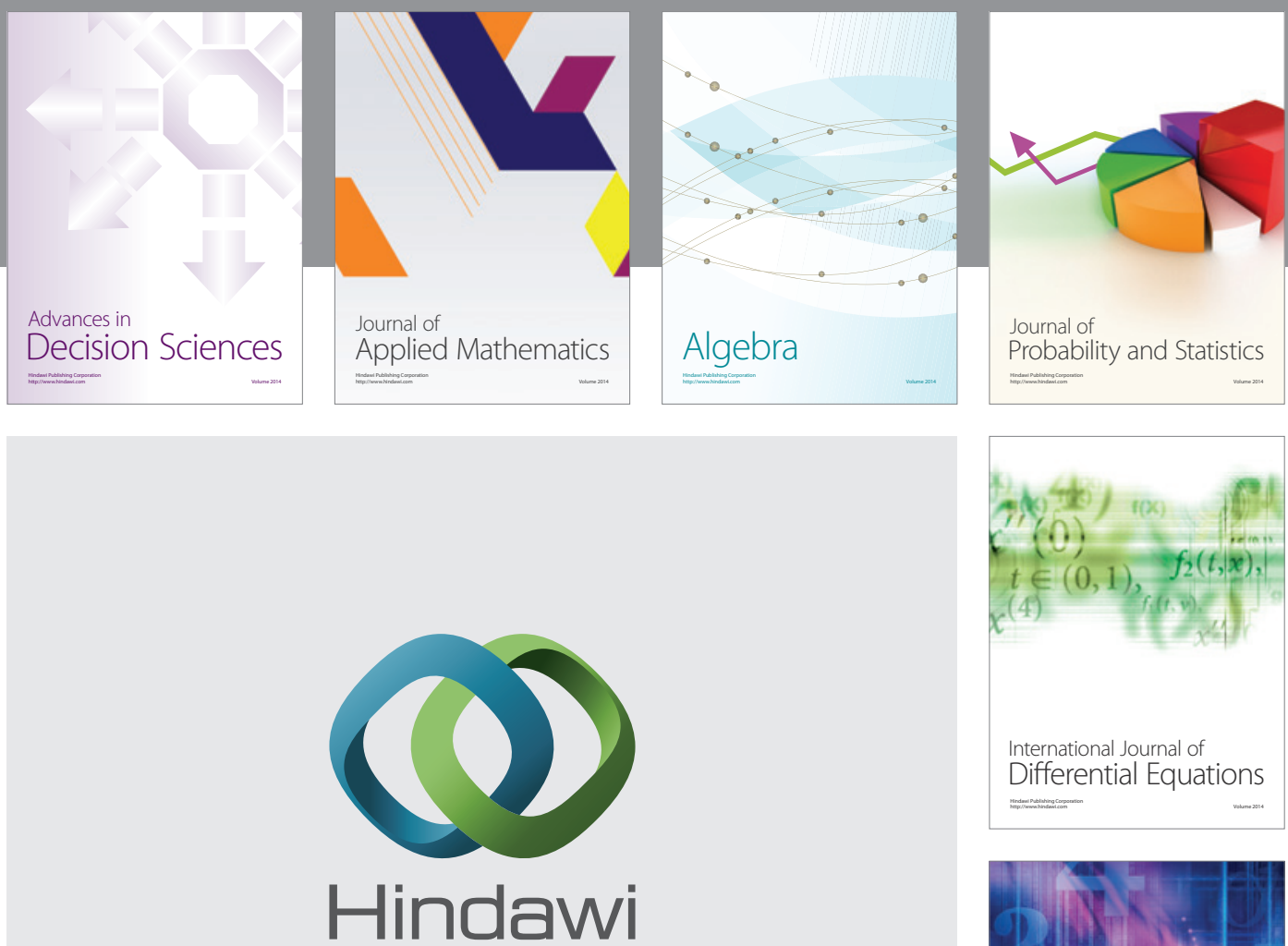

Submit your manuscripts at http://www.hindawi.com
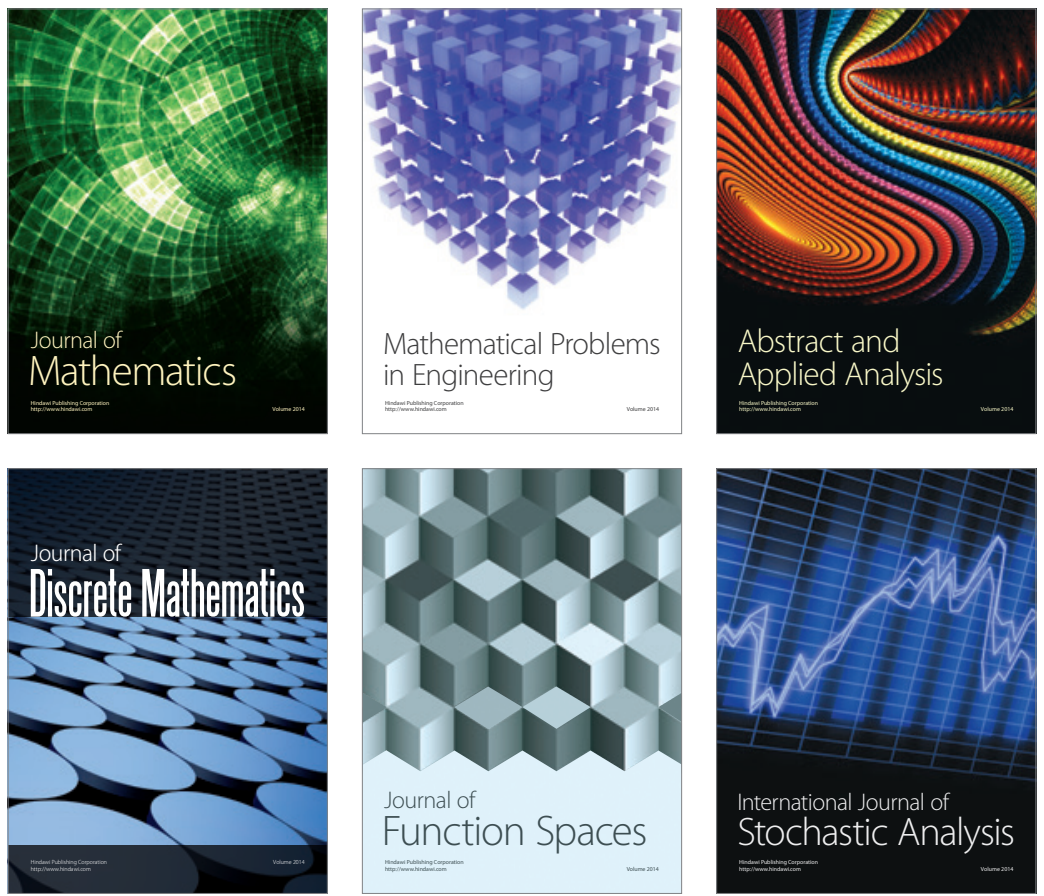

Journal of

Function Spaces

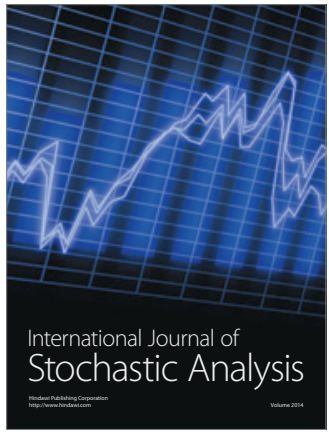

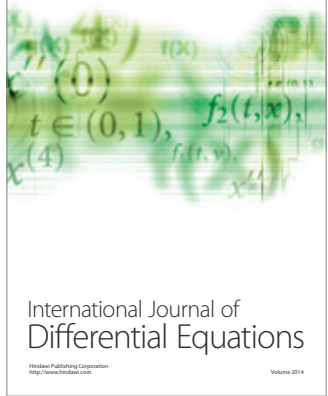
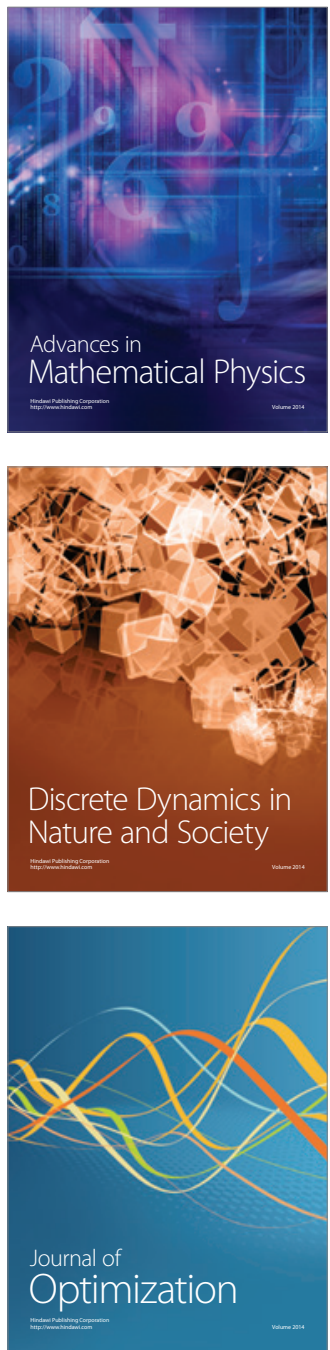